\title{
Rasgrf2 controls dopaminergic adaptations to alcohol in mice
}

\author{
Alanna C. Easton ${ }^{1 *}$, Andrea Rotter ${ }^{2 \star}$, Anbarasu Lourdusamy ${ }^{1}$, Sylvane Desrivières ${ }^{1}$, \\ Alberto Fernández-Medarde ${ }^{3}$, Teresa Biermann ${ }^{2}$, Cathy Fernandes ${ }^{1}$, Eugenio \\ Santos $^{3}$, Johannes Kornhuber ${ }^{2}$, Gunter Schumann ${ }^{1}$, Christian P. Müller ${ }^{1,2}$ \\ ${ }^{1}$ MRC Social, Genetic and Developmental Psychiatry Centre, Institute of Psychiatry, King's College \\ London, De Crespigny Park, London SE5 8AF, UK \\ ${ }^{2}$ Department of Psychiatry and Psychotherapy, University Clinic, Friedrich-Alexander-University \\ Erlangen-Nuremberg, Schwabachanlage 6, 91054 Erlangen, Germany \\ ${ }^{3}$ CIC-IBMCC, University of Salamanca - CSIC, Spain;
}

* These authors contributed equally to the paper

Corresponding author: $\quad$ Christian P. Müller Section of Addiction Medicine Department of Psychiatry and Psychotherapy Friedrich-Alexander-University Erlangen-Nuremberg Schwabachanlage 6

Erlangen 91054

Phone: +49 (0) 91318536896

Email: Christian.Mueller@uk-erlangen.de 


\section{Abstract}

Alcohol abuse leads to serious health problems with no effective treatment available. Recent evidence suggests a role for ras-specific guanine-nucleotide releasing factor 2 (RASGRF2) in alcoholism. Rasgrf2 is a MAPK/ERK activating protein, which has been linked to neurotransmitter release and monoaminergic receptor adaptations. Rasgrf2 knock out (KO) mice do not develop a dopamine response in the nucleus accumbens after an alcohol challenge and show a reduced consumption of alcohol. The present study aims to further characterize the role of Rasgrf2 in dopaminergic activation beyond the nucleus accumbens following alcohol treatment. Using in-vivo microdialysis we found that alcohol increases dopamine levels in the dorsal striatum. This increase was absent in Rasgrf2 KO mice. There was no difference in expression of dopamine transporter (DAT), dopamine receptor regulating factor (DRRF), or dopamine D2 receptor (DRD2) mRNA in the brain between Rasgrf2 KO and WT mice. After sub-chronic alcohol treatment, DAT and DRRF, but not DRD2 mRNA expression in Rasgrf2 KO mice decreased relative to WT levels. Brain adaptations were positively correlated with blood expression levels. These data suggest a Rasgrf2 controls dopaminergic signaling and adaptations to alcohol also beyond the nucleus accumbens.

\section{Keywords:}

Rasgrf2, dopamine, alcohol, microdialysis, dopamine transporter, dopamine receptor regulating factor, D2 receptor 


\section{Introduction}

Alcohol use develops into addiction in a significant number of people. Alcohol abuse leads to serious health problems in approximately $10-20 \%$ of chronic alcohol consumers and results in 2.5 million deaths each year [1]. Although significant heritability estimates of alcohol addiction have been identified [2], underlying genetic influences are not surfacing as quickly $[3,4]$. Recent evidence suggests a role of the ras-specific guanine-nucleotide releasing factor 2 (RASGRF2) gene in alcoholism [5]. The study reports RASGRF2 in the regulation of mesolimbic dopamine activity which ultimately impacts upon reward response and alcohol consumption.

Rasgrf2 is enriched at synaptic sites of the CNS. It is a calmodulin (CaM) associated guanine nucleotide exchange factor and becomes active in the presence of CaM. Activation of Rasgrf2 in turn activates the RAS protein which is known to be involved in the MAPK/ERK signal transduction pathway [6] and the regulation of signalling pathways in cells. Rasgrf2, as a MAPK/ERK activating protein, has therefore been linked to neurotransmitter release [7]. The RAS-MAPK/ERK pathway has also been linked to dopamine receptors $[8,9)$, and has been isolated as a binding partner of the dopamine transporter (DAT; [10]). These studies report a role for Rasgrf2 both pre- and post-synaptically, indicating a preferential role of Rasgrf2 in the regulation of synaptic transmission and plasticity.

RASGRF2 has recently been implicated as a novel candidate in the regulation of alcohol consumption via dopaminergic signalling in the mesolimbic system [5]. A genome wide analysis of 28,188 people found polymorphisms of RASGRF2 to be significantly associated with alcohol consumption. The same study reported that Rasgrf2 knock-out (KO) mice consume significantly less alcohol and have a reduced alcohol preference compared to their wild-type (WT) counterparts. Rasgrf2 KO mice 
do not experience dopamine increases in the nucleus accumbens (ventral striatum) after an alcohol challenge compared to wild type (WT) mice, suggesting that Rasgrf2 $\mathrm{KO}$ mice do not experience the motivationally rewarding properties of alcohol to the same extent as WT mice [5].

Previous studies have shown no difference in Rasgrf2 expression up to six hours following acute cocaine [11] and amphetamine [12] administration at several doses in the ventral- and dorsal striatum, as well as in other areas of the forebrain including the medial prefrontal cortex and the hippocampus. Both cocaine and amphetamine did however produce the behavioural activating effects typically associated with these drugs such as increased locomotion and sniffing and biting behaviours $[11,12]$. These studies also report alterations in Rasgrf1 expression in these regions, highlighting the heterogeneity of these two isoforms in synaptic plasticity. Acute injection of cocaine [13-17] and amphetamine [14,15,18-20] increase phosphorylation of ERKs in the striatum. In contrast, moderate blood alcohol levels reduce ERK phosphorylation in brain regions including the amygdala, cerebellum, hippocampus, frontal cortex and dorsal striatum. However, during alcohol withdrawal increases in ERK phosphorylation have been reported [21]. The present study aimed to further investigate the role Rasgrf2 in the regulation of dopaminergic activity after alcohol treatment. 


\section{Material and Methods}

All housing and experimental procedures were performed in accordance with the U.K. Home Office Animals (Experimental Procedures) Act 1986.

\subsection{Animals}

Male WT (+/+) and null mutant (-/- KO) Rasgrf2 ${ }^{\text {tm1Esn }}$ [22] mice were studied. Mice were generated using a gene targeting strategy and subsequently kept on a genetic background of 129/S-J and C57BL/6, detailed methods as previously described [22]. The method inactivates the Grf2 locus by targeting its CDC25- $\mathrm{H}$ catalytic domain, thereby disrupting the guanine nucleotide exchange factor (GEF) activity of Rasgrf2. Animals were individually housed, provided with food and water ad libitum, and kept on a 12:12 hour light: dark cycle (lights on at $7.00 \mathrm{am}$ ). Experiments were performed during the light cycle between 09:00 and 16:00 h, in a pseudorandom order. Room temperature was maintained between $19^{\circ} \mathrm{C}$ and $22^{\circ} \mathrm{C}$ at a humidity of $55 \%( \pm 10 \%)$. Animals were housed in Tecniplast cages $(32 \mathrm{~cm} \times 16 \mathrm{~cm} \times 14 \mathrm{~cm})$, using Litaspen sawdust and nesting materials, (Sizzlenest, Datsand, Manchester UK).

\subsection{In vivo microdialysis}

Mice were deeply anaesthetised (detailed methods as previously described [23]) and a guide cannula (Microbiotech/se AB, Stockholm, Sweden) was aimed at the DStr $\left(\mathrm{A}:+0.5 ; \mathrm{L}: \pm 2.3 ; \mathrm{V}:-2.4\right.$ angle $\pm 10^{\circ}$ from midline) using coordinates relative to bregma [24]. On the day of the experiment, a microdialysis probe of a concentric design, membrane length of $2 \mathrm{~mm}$ (MAB 6.14.2.), was inserted into the guide cannula under a short (3-5min) Isoflurane anaesthesia $\left(\mathrm{O}_{2}\right.$ at $1 \mathrm{~L} / \mathrm{min}$, Isoflurane at $3 \%$ to induce and $2 \%$ to sustain). Samples were collected every 20 minutes. Three 
samples were taken during the first testing hour of the experiment to measure baseline quantities of DA [25]. An injection of alcohol was then administered i.p. $\left(2 \mathrm{~g} / \mathrm{kg}, V_{\mathrm{inj}}=10 \mathrm{ml} / \mathrm{kg}\right)$. A further nine samples were collected. All samples were analysed using HPLC-ED (as described $[25,26]$ ) to measure DA levels in response to alcohol administration. Alcohol naïve male mice were used for this test (Rasgrf2 KO: n=8; Rasgrf2 WT: n=8). In-vivo microdialysis was carried out at least 5 days after surgery on day one of the experiment during which animals received an alcohol injection ( $2 \mathrm{~g} / \mathrm{kg}$, i.p.). This provided an acute neurochemical response to alcohol treatment. Once microdialysis experiments were complete, animals were sacrificed by cervical dislocation. Brains were fixed in $4 \%$ formaldehyde solution and stored at $4{ }^{\circ} \mathrm{C}$. Brains were sliced on a microtome and stained with cresyl violet for analysis of probe placement. Only animals with probe placement within the DStr were considered for data analysis. Since all probes were surgically inserted accurately within the DStr, all animals were considered for data analysis.

\subsection{Gene Expression}

In order to investigate whether expression of dopaminergic genes involves Rasgrf2, we measured mRNA expression profiles of the dopamine transporter (DAT), dopamine receptor regulating factor (DRRF), and dopamine D2 receptor (DRD2) in the frontal cortex (FC), ventral striatum (VStr), dorsal striatum (DStr), hippocampus (Hipp), and the cerebellum (Cer) of Rasgrf2 KO and WT mice after sub-chronic treatment with either saline or alcohol. In order to test whether peripheral (blood) gene expression would be predictive for brain adaptations, we also measured mRNA expression in the blood and performed a correlation analysis with specific brain areas. Single housed Rasgrf2 KO and WT mice ( $n=8-9 /$ group) received a daily i.p. 
injection with either alcohol $(3.5 \mathrm{~g} / \mathrm{kg}$ ) or saline for 7 days. After each injection animals were placed back to their home cages. 120 minutes after the last injection, animals were sacrificed by cervical dislocation and the brain and spleen were removed and immediately cooled on dry ice. Tissue was kept at -80 degree until further analysis. The frozen brain was placed on a cold dissecting surface and it was hemisected by cutting in the sagittal plane. Brain areas were dissected according to Paxinos and Franklin [27]. As previously described [28], total RNA was extracted using a modified Qiagen-protocol: A phenol-extraction in Qiazol (Qiagen) was followed by column-purification with Rneasy Mini Kit (Qiagen), including DNase digestion according to manufacturer's protocol. cDNA was synthesized using iScript cDNA Synthesis Kit (Biorad, Munich/Germany) following manufacturer's instructions. Quantitative rt-PCR was performed using SYBR Green I Master Mix buffer (Applied Biosystems), and reactions were run on an iCycler (Roche) using a three-step standard protocol. The annealing temperature was optimized for all primer pairs and ranged from 55 to $65 \mathrm{C}^{\circ}$. PCR products were visualized on standard $2 \%$ agarose gels with ethidium bromide to eliminate the possibility of having amplified genomic DNA. beta-Actin was used as internal standard, and CT values were calculated from differences between beta-Actin and the target genes. All experiments were repeated at least three times and the mean value used for further analysis.

The following primer pairs were used:

B-Actin-F: CTGTATTCCCCTCCATCGTG

B-Actin-R: GAAGATCTGGCACCACACCT

DRD2-F: TGGACTCAACAACACAGACCAGAATG

DRD2-R: GATATAGACCAGCAGGGTGACGATGA 
DRRF-F: CAAAAGCCACCGCTGTCCCTTC

DRRF-R: GGCGAACTTCTTGTCACAGCCA

DAT-F: ATCAACCCACCGCAGACACCAGT

DAT-R: CTTTCAGGACAGGGCAG

\subsection{Statistical Analysis}

All graphical output data is expressed as a mean \pm SEM. Microdialysis: Baseline neurochemical data were analysed using one way ANOVA. Alcohol induced neurochemical effects were expressed as a percentage of the mean of the three baseline samples which were taken as $100 \%$ to standardise the data across groups. Data were compared using two-way ANOVA with factors genotype (2) and time (13). To compare alcohol effects of certain time points, pre-planned t-test point-by-point comparisons were performed. Gene expression: The mRNA expression was calculated as deltaCT (mean+SEM). For statistical analysis, two-way ANOVAs with factors genotype (2) and treatment (2) were used. To evaluate genotype effects on single brain area and blood level, planned comparisons using Bonferroni-corrected Fisher's LSD test were calculated. A correlation analysis of brain and blood mRNA expression (Pearson correlation) was performed for all target mRNA with pooled genotypes. The software used was Statistica 7.0. A significance level of $p<0.05$ was used to test for statistical significance. 


\section{Results}

\subsection{No alcohol-induced dopamine response in the dorsal striatum of Rasgrf2 KO mice}

Resting DA levels in alcohol naïve Rasgrf2 KO and WT mice were comparable between groups in the DStr $\left(F_{1,38}=0.06, p=0.80\right.$; Fig. $\left.1 A\right)$. An acute alcohol treatment increase extracellular DA levels in WT animals. This effect was absent in Rasgrf KO mice. A two-way ANOVA showed significant effects of time $\left(F_{12,84}=2.71, p=0.004\right)$ and genotype $\left(F_{1,7}=6.63, p=0.04\right)$, but no significant interaction $\left(F_{12,84}=1.37, p=0.20\right.$;

Fig 1B). Wild type DA levels in response to an alcohol injection were significantly different from Rasgrf2 KO mice $120 \min (p=0.04)$ and 140 min $(p=0.04)$ after administration (Fig. 1B).

\subsection{Alcohol treatment reduced DAT and DRRF expression in several brain regions of Rasgrf2 $\mathrm{KO}$ mice}

There was no difference in DAT mRNA expression between Rasgrf2 KO and WT mice after saline treatment across all brain areas (Fig 2A). After alcohol treatment DAT mRNA expression in Rasgrf2 $\mathrm{KO}$ mice decreased relative to WT mRNA expression levels (higher deltaCT; Fig 2B). There was a significant effect of genotype in the $F C\left(F_{1,34}=4.06, p=0.05\right)$, but not of treatment or interaction $(p>0.05)$. In the VStr, there was a significant genotype effect $\left(F_{1,34}=5.49, p<0.025\right)$, but no significant effects of treatment or of interaction $(p>0.05)$. In the DStr there were no significant effects ( $p>0.05)$. There was a significant effect of genotype $\left(F_{1,34}=17.41\right.$, $p=0.0002)$, treatment $\left(F_{1,34}=20.06, p=0.00008\right)$, and interaction $\left(F_{1,34}=6.20\right.$, $\mathrm{p}=0.0178$ ) in the Hipp. In the Cer, there was a significant effect of genotype $\left(F_{1,34}=5.69, p=0.023\right)$ and an interaction $\left(F_{1,34}=6.02, p=0.019\right)$, but no significant 
treatment effect $(p>0.05)$. Post hoc Fisher's LSD tests revealed a significant difference between Rasgrf2 $\mathrm{KO}$ and WT mice after alcohol treatment in the FC $(p=0.03)$, VStr $(p=0.026)$, Hipp $(p<0.0001)$, and Cer $(p=0.0026)$, but not in the DStr $(p>0.05)$.

There was no difference in DRRF mRNA expression between Rasgrf2 KO and WT mice after saline treatment across all brain areas (Fig 2C). After alcohol treatment, DRRF mRNA expression in Rasgrf2 $\mathrm{KO}$ mice decreased relative to WT mRNA expression levels (higher deltaCT; Fig 2D). There was a significant effect of genotype in the $F C\left(F_{1,34}=5.90, p<0.0205\right)$, but not of treatment or interaction $(p>0.05)$. In the VStr, there was a significant genotype $\left(F_{1,34}=12.60, p<0.0012\right)$ and treatment effect $\left(F_{1,34}=5.38, p=0.0265\right)$, but no significant interaction $(p>0.05)$. In the DStr, there was a significant genotype effect $\left(F_{1,34}=4.42, p=0.04\right)$, but not of treatment or interaction $(p>0.05)$. There were no significant effects in the Hipp $(p>0.05)$. In the Cer, there was a significant effect of genotype only $\left(F_{1,34}=6.80\right.$, $\mathrm{p}=0.0134$ ). Post hoc Fisher's LSD tests revealed a significant difference between Rasgrf2 KO and WT mice after alcohol treatment in the FC $(p=0.024)$, VStr $(p=0.0003)$, DStr $(p=0.0192)$, and Cer $(p=0.0168)$, but not in the Hipp $(p>0.05)$.

There were no genotype differences in DRD2 mRNA expression after either saline (Fig 2E) or alcohol (Fig 2F) treatments across all brain areas measured in the present study ( $p>0.05)$. Levels of DAT, DRRF and DRD2 in the blood after both a saline injection and an alcohol injection were not significantly different between Rasgrf2 KO and WT mice. 


\subsection{Correlations in the mRNA expression profiles}

Co-expression analysis showed that there was a positive relationship in DAT mRNA expression between specific brain areas and blood. A strong correlation was seen between spleen vs. FC $(r=0.75 ; p<0.0001 ;$ Fig $3 A)$ and spleen vs. Cer $(r=0.77$; p<0.0001; Fig 3E). A significant correlation was also found for spleen vs. VStr $(r=0.49 ; p=0.002$; Fig 3B), spleen vs. DStr $(r=0.52 ; p=0.001 ;$ Fig $3 C)$, and spleen vs. Hipp ( $r=0.44 ; p=0.005 ;$ Fig 3D). FC and blood expression of DRRF was also positively correlated, although the nature of this relationship was rather weak $(r=0.33 ; p=0.042 ;$ Fig 3F). DRD2 mRNA expression was also positively correlated between brain and blood. There was a relationship seen spleen vs. FC ( $r=0.44$; $p=0.006$; Fig 3G), spleen vs. VStr ( $r=0.42 ; p=0.009 ;$ Fig $3 H)$, spleen vs. Hipp $(r=0.47$; $p=0.003$; Fig 3I), and between spleen vs. Cer $(r=0.60 ; p<0.0001 ;$ Fig 3J). 


\section{Discussion}

In order to experience the acute reinforcing effects of alcohol, several neurotransmitter systems in the reward centres of the brain are recruited $[29,30]$. Changes in DA levels in the NAcc in response to an alcohol challenge, is an indicator of the incentive properties of alcohol [31,32]. Previous work suggests that Rasgrf2 is not implicated in the striatum following acute drug action of cocaine [11] and amphetamine [12] exposure. However, the absence of an alcohol-induced DA increase in the NAcc of Rasgrf2 KO mice may contribute towards a diminished alcohol consumption and preference [5]. Rasgrf2 has been shown to mediate these responses via the dopaminergic neurons of the ventral tegmental area [5]. In addition, polymorphisms in the human RASGRF2 gene have been associated with alcohol intake [5] and changes in brain structure, including the medial prefrontal cortex, in an elderly human population [33]. The present study aimed to further characterize how Rasgrf2 regulates dopaminergic mechanisms in the DStr in response to alcohol administration. In contrast to the NAcc [5], present data revealed no differences in DA baseline levels between Rasgrf2 KO and WT mice in the DStr. When challenged with alcohol, extracellular DA levels in the DStr increased in WT mice, but remained at basal levels in the Rasgrf $2 \mathrm{KO}$ mice. These data complement previous research by Stacey and colleagues [5] which revealed the absence of a DA response in Rasgrf2 KO mice in the NAcc. Together, past and present data suggest that aberrant DA activity in the ventral striatum, in particular the Nacc and dorsal striatum of Rasgrf2 KO mice may account for the abated motivational and rewarding effects of alcohol. This acute effect may impact upon subsequent alcohol consumption and preference behaviours in these mice. 
Rasgrf2 has been isolated as a binding partner of the DAT [10], the primary mechanism for synaptic DA clearance in the striatum. The RAS-MAPK/ERK pathway has also been associated with dopamine receptors $[8,9]$. These studies suggest an important role for Rasgrf2 at the synapse in the regulation of reinforcement via dopaminergic signalling [10]. Brain expression analysis indicated no differences in DAT, DRRF or DRD2 between Rasgrf2 $\mathrm{KO}$ and WT mice after a saline injection, indicating that Rasgrf2 has no role in the control of DAT, DRRF, and DRD2 expression under basal conditions. After sub-chronic alcohol administration, there was no difference between genotypes in DRD2 mRNA expression levels. However, DAT and DRRF mRNA expression decreased in Rasgrf2 $\mathrm{KO}$ relative to WT mice. Rasgrf2 is partially responsible for the regulation of DAT [10]. Decreased DAT expression in the FC, VStr, Hipp, and Cer of the Rasgrf2 $\mathrm{KO}$ mice in the present study would suggest a reduced DA reuptake from the synaptic cleft, and thus enhanced extracellular DA levels in comparison to WT mice in these areas. This view is supported by previous microdialysis data from Stacey and colleagues [5] which showed enhanced DA levels in the Nacc of Rasgrf2 mice at basal conditions and a reduced DA response after an alcohol challenge. These findings also suggest that basal DA levels appear to be under different Rasgrf2-DAT mediated control in the DStr vs. VStr.

Significant decreases in DRRF expression in Rasgrf2 $\mathrm{KO}$ mice were seen in the FC, VStr, DStr, and Cer compared to WT mice in response to an alcohol challenge. DRRF is a transcription factor and regulates transcription for a number of different promoters. DRRF is important for modulating dopaminergic transmission in the brain and can alter DA receptor expression [34]. DRRF is known to regulate the DRD2 gene as well as the DRD1A and DRD3 promoters [34]. Acute cocaine administration 
has been shown to produce a large extracellular DA increase which in turn produces a decrease in DRRF mRNA levels in the NAcc core and shell [35]. Conversely, caffeine produces a much smaller increase in DA levels in the striatum and does not alter DRRF mRNA [35]. These data are in keeping with the suggested role of DRRF as a DA receptor regulator [36]. DRRF has the potential to be a contributing mechanism in the DStr in the mediation of DA concentration in the synaptic cleft following acute alcohol administration in the present study. We also quantified DRD2 mRNA expression in the DStr, but there were no significant differences between Rasgrf2 $\mathrm{KO}$ and WT mice. This suggests that DRRF does not alter DRD2 expression, but may act via DRD1 or DRD3 instead. The RAS-MAPK/ERK pathway has also been associated with DRD1 $[8,9]$ and could be the mediating mechanism in the current study. In addition, alcohol-induced adaptations in the noradrenergic and serotonergic systems appear under the control of Rasgrf2, which may also contribute to the observed phenotype [37].

Co-expression analysis indicated a positive relationship in DAT mRNA expression between all brain areas and blood, with particularly strong correlations in the $\mathrm{FC}$ and Cer. The nature of the correlation between blood and brain FC DRRF was positive although a rather weak association. DRRF showed no correlation in either the DStr or VStr. Expression of DRD2 showed a weak but significant correlation between blood and brain in all areas except the DStr. This may help to predict mRNA expression changes in response to alcohol administration in the brain by using respective markers in the blood.

\section{Conclusions}


Present data provide further evidence for a distinct contribution of Rasgrf2 in dopaminergic signaling in the dorsal striatum and suggests that Rasgrf2 may mediate dopaminergic adaptations to alcohol in mice.

\section{Aknowledegements}

This work was supported by the Biomedical Research Council (U.K.) and funds of the Friedrich-Alexander-University Erlangen-Nuremberg. A.E. is a recipient of a studentship from the Medical Research Council, U.K. 


\section{Figure Legends}

Figure 1: Extracellular dopamine (DA) levels in the dorsal striatum (DStr) of Rasgrf2 knock-out (KO) and wild-type (WT) mice. (A) Baseline DA levels prior to alcohol treatment. (B) DA levels in response to an alcohol challenge. The arrow indicates the time of alcohol treatment $\left({ }^{\star} p<0.05\right.$, WT vs. Rasgrf2 KO).

Figure 2: The effects of alcohol treatment (3.5 g/kg, i.p., 7d) on mRNA expression in the brain of Rasgrf2 knock-out (KO) and wild-type (WT) mice (DAT- dopamine transporter, DRRF-dopamine receptor regulating factor, DRD2- dopamine D2 receptor; FC-frontal cortex, VStr-ventral striatum, DStr-dorsal striatum, Hipphippocampus, Cer-cerebellum; $\left.{ }^{*} p<0.05,{ }^{* *} p<0.01,{ }^{* * *} p<0.001\right)$.

Figure 3: The blood mRNA expression correlates with brain area expression in Rasgrf2 knock out and wild type mice after alcohol (3.5 g/kg, i.p., 7d) and saline treatment. Data pooled for genotype and treatment (DAT- dopamine transporter, DRRF-dopamine receptor regulating factor, DRD2 - dopamine D2 receptor). 


\section{References}

[1] WHO. World Health Organisation Global Status Report on Alcohol and Health 2011.

[2] Plomin R. The role of inheritance in behavior. Science. 1990;248:183-8.

[3] Stacey D, Clarke TK, Schumann G. The genetics of alcoholism. Curr Psychiatry Rep. 2009;11:364-9.

[4] Schumann G, Coin LJ, Lourdusamy A, Charoen P, Berger KH, Stacey D, et al. Genome-wide association and genetic functional studies identify autism susceptibility candidate 2 gene (AUTS2) in the regulation of alcohol consumption. Proc Natl Acad Sci U S A. 2011;108:7119-24.

[5] Stacey D, Bilbao A, Maroteaux M, Jia T, Easton AC, Longueville S, et al. RASGRF2 regulates alcohol-induced reinforcement by influencing mesolimbic dopamine neuron activity and dopamine release. Proc Natl Acad Sci U S A. 2012;109:21128-33.

[6] Agell N, Bachs O, Rocamora N, Villalonga P. Modulation of the Ras/Raf/MEK/ERK pathway by $\mathrm{Ca}(2+)$, and calmodulin. Cell Signal. $2002 ; 14: 649-54$

[7] Bloch-Shilderman E, Jiang H, Abu-Raya S, Linial M, Lazarovici P. Involvement of extracellular signal-regulated kinase (ERK) in pardaxin-induced dopamine release from PC12 cells. J Pharmacol Exp Ther. 2001;296:704-11.

[8] Tian X, Gotoh T, Tsuji K, Lo EH, Huang S, Feig LA. Developmentally regulated role for Ras-GRFs in coupling NMDA glutamate receptors to Ras, Erk and CREB. EMBO J. 2004;23:1567-75.

[9] Girault JA, Valjent E, Caboche J, Herve D. ERK2: a logical AND gate critical for drug-induced plasticity? Curr Opin Pharmacol. 2007;7:77-85. 
[10] Maiya R, Ponomarev I, Linse KD, Harris RA, Mayfield RD. Defining the dopamine transporter proteome by convergent biochemical and in silico analyses. Genes Brain Behav. 2007;6:97-106.

[11] Zhang GC, Hoffmann J, Parelkar NK, Liu XY, Mao LM, Fibuch EE, et al. Cocaine increases Ras-guanine nucleotide-releasing factor 1 protein expression in the rat striatum in vivo. Neurosci Lett. 2007;427:117-21.

[12] Parelkar NK, Jiang Q, Chu XP, Guo ML, Mao LM, Wang JQ. Amphetamine alters Ras-guanine nucleotide-releasing factor expression in the rat striatum in vivo. Eur J Pharmacol. 2009;619:50-6.

[13] Valjent E, Corvol JC, Pages C, Besson MJ, Maldonado R, Caboche J. Involvement of the extracellular signal-regulated kinase cascade for cocainerewarding properties. J Neurosci. 2000;20:8701-9.

[14] Valjent E, Pascoli V, Svenningsson P, Paul S, Enslen H, Corvol JC, et al. Regulation of a protein phosphatase cascade allows convergent dopamine and glutamate signals to activate ERK in the striatum. Proc Natl Acad Sci U S A. 2005;102:491-6.

[15] Valjent E, Corvol JC, Trzaskos JM, Girault JA, Herve D. Role of the ERK pathway in psychostimulant-induced locomotor sensitization. BMC neuroscience. $2006 ; 7: 20$.

[16] Zhang L, Lou D, Jiao H, Zhang D, Wang X, Xia Y, et al. Cocaine-induced intracellular signaling and gene expression are oppositely regulated by the dopamine D1 and D3 receptors. J Neurosci. 2004;24:3344-54.

[17] Jenab S, Festa ED, Nazarian A, Wu HB, Sun WL, Hazim R, et al. Cocaine induction of ERK proteins in dorsal striatum of Fischer rats. Brain Res Mol Brain Res. 2005;142:134-8. 
[18] Choe ES, Chung KT, Mao L, Wang JQ. Amphetamine increases phosphorylation of extracellular signal-regulated kinase and transcription factors in the rat striatum via group I metabotropic glutamate receptors. Neuropsychopharmacology. 2002;27:565-75.

[19] Choe ES, Wang JQ. CaMKII regulates amphetamine-induced ERK1/2 phosphorylation in striatal neurons. Neuroreport. 2002;13:1013-6.

[20] Valjent E, Pages C, Herve D, Girault JA, Caboche J. Addictive and nonaddictive drugs induce distinct and specific patterns of ERK activation in mouse brain. Eur J Neurosci. 2004;19:1826-36.

[21] Sanna PP, Simpson C, Lutjens R, Koob G. ERK regulation in chronic ethanol exposure and withdrawal. Brain Res. 2002;948:186-91.

[22] Fernández-Medarde A, Esteban LM, Núñez A, Porteros A, Tessarollo L, Santos E. Targeted disruption of Ras-Grf2 shows its dispensability for mouse growth and development. Mol Cell Biol. 2002;22:2498-504.

[23] Easton AC, Lucchesi W, Lourdusamy A, Lenz B, Solati J, Golub Y, et al. alphaCaMKII Autophosphorylation Controls the Establishment of Alcohol Drinking Behavior. Neuropsychopharmacology. 2013; 38:1636-1647.

[24] Franklin KBJ, Paxinos G. The mouse brain in stereotaxic coordinates. 2nd ed. San Diego: Academic Press; 1997.

[25] Pum ME, Huston JP, De Souza Silva MA, Müller CP. Visual sensory-motor gating by serotonin activation in the medial prefrontal and occipital, but not in the rhinal, cortices in rats. Neuroscience. 2008;153:361-72.

[26] Pum M, Carey RJ, Huston JP, Müller CP. Dissociating effects of cocaine and damphetamine on dopamine and serotonin in the perirhinal, entorhinal, and 
prefrontal cortex of freely moving rats. Psychopharmacology (Berl). 2007;193:375-90.

[27] Paxinos G, Franklin KBJ. The Mouse Brain in Stereotaxic Coordinates. 2nd ed: Academic Press; 2003.

[28] Rotter A, Biermann T, Amato D, Schumann G, Desrivieres S, Kornhuber J, et al. Glucocorticoid receptor antagonism blocks ethanol-induced place preference learning in mice and attenuates dopamine D2 receptor adaptation in the frontal cortex. Brain Res Bull. 2012;88:519-24.

[29] Koob GF, Roberts AJ, Schulteis G, Parsons LH, Heyser CJ, Hyytia P, et al. Neurocircuitry targets in ethanol reward and dependence. Alcohol Clin Exp Res. $1998 ; 22: 3-9$.

[30] Müller CP, Homberg J, The role of serotonin in drug use and addiction, Behav Brain Res. 2014, in press.

[31] Di Chiara G, Imperato A. Drugs abused by humans preferentially increase synaptic dopamine concentrations in the mesolimbic system of freely moving rats. Proc Natl Acad Sci U S A. 1988;85:5274-8.

[32] Koob GF. Drugs of abuse: anatomy, pharmacology and function of reward pathways. Trends Pharmacol Sci. 1992;13:177-84.

[33] Roussotte FF, Gutman BA, Hibar DP, Jahanshad N, Madsen SK, Jack CR, Jr., et al. A single nucleotide polymorphism associated with reduced alcohol intake in the RASGRF2 gene predicts larger cortical volumes but faster longitudinal ventricular expansion in the elderly. Frontiers in Aging Neuroscience. 2013;5:93.

[34] Hwang CK, D'Souza UM, Eisch AJ, Yajima S, Lammers CH, Yang Y, et al. Dopamine receptor regulating factor, DRRF: a zinc finger transcription factor. Proc Natl Acad Sci U S A. 2001;98:7558-63. 
[35] Di Chiara G. Drug addiction as dopamine-dependent associative learning disorder. Eur J Pharmacol. 1999;375:13-30.

[36] Spyraki C, Sealfon SC. Regulation of dopamine D2 receptor mRNA expression in the olfactory tubercle by cocaine. Brain Res Mol Brain Res. 1993;19:313-7.

[37] Easton AC, Rotter A, Lourdusamy A, Desrivieres S, Fernández-Medarde A, Biermann T, Fernandes C, Santos E, Kornhuber J, Schumann G, Müller CP. Rasgrf2 controls noradrenergic involvement in the acute and subchronic effects of alcohol in the brain. Psychopharmacology. 2014, in press. 